\title{
ON A FOURTH ORDER EQUATION IN 3-D
}

\author{
XINGWAng $\mathrm{XU}^{1, *}$ And PaUl C. YANG ${ }^{2, \dagger}$
}

\begin{abstract}
In this article we study the positivity of the 4-th order Paneitz operator for closed 3-manifolds. We prove that the connected sum of two such 3-manifold retains the same positivity property. We also solve the analogue of the Yamabe equation for such a manifold.
\end{abstract}

Mathematics Subject Classification. 53C21, 35G20.

Received January 14, 2002.

\section{INTRODUCTION}

In the analytic study of conformal structures in dimensions greater than two, it is fruitful to consider the family of $Q$-curvature equations as natural generalization of the Yamabe equation. Since the work of Paneitz [9] there has been a number of such equations introduced by Branson [2] and Fefferman and Graham [7].

In a series of papers $[3,4]$ it is demonstrated that solutions of these equations lead to significant results for nonlinear analysis as well as for conformal geometry in dimension four. A number of authors have investigated these equations in dimensions higher than four, for example Djadli et al. [6], Hebey and Robert [8] and Ahmedou et al. [1]. In this paper, we call attention to the validity of the equation in dimension three and begin a preliminary investigation of the fourth order Paneitz equation in the most favorable situation. Let us recall the Paneitz operator

$$
P=(-\Delta)^{2}+\delta\left(\frac{5}{4} R g-4 R i c\right) d-\frac{1}{2} Q
$$

where

$$
Q=-2|R i c|^{2}+\frac{23}{32} R^{2}-\frac{1}{4} \Delta R
$$

Under a conformal change of metrics $\bar{g}=u^{-4} g$ with $u>0$, the Paneitz operator enjoys the following conformal covariance property:

$$
\bar{P} w=u^{7} P(u w) .
$$

Keywords and phrases: Paneitz operator, conformal invariance, Sobolev inequality, connected sum.

${ }^{1}$ Department of Mathematics, National University of Singapore, 2 Science Drive 2, 119260 Singapore; e-mail: matxuxw@nus . edu . sg

* Research of Xu supported by NUS Research Grant R-146-000-033-112.

2 Department of Mathematics, Princeton University, Princeton, NJ 08544-1000 U.S.A.; e-mail: yang@math.princeton.edu

$\dagger$ Research of Yang supported by NSF DMS-0070526. 
In fact the $Q$-curvatures are related by the nonlinear equation

$$
P u=-\frac{1}{2} Q_{\bar{g}} u^{-7}
$$

In this article, we investigate the conformal classes for which the Paneitz operator is positive. In a previous article [10], we have shown that positivity is preserved under the connected sum operation in dimensions greater than four. While we do not know if the same property continues to hold in dimension four, we will show here that it remains true in dimension three.

Theorem 1.1. Let $(M, g)$ and $\left(M^{\prime}, g^{\prime}\right)$ be closed 3-manifolds on which the Paneitz operator is positive, then there exists a conformal structure on the connected sum $M \# M^{\prime}$ for which the Paneitz operator is positive.

The basic reason underlying this result is that on the cylinder, the energy integrand

$$
e(u)=\left[(\Delta u)^{2}+\left(\frac{5}{4} R g-4 R c\right)(\nabla u, \nabla u)-\frac{1}{2} Q u^{2}\right] \mathrm{d} V
$$

for the Paneitz operator becomes positive after integrating over the 2-spheres.

The proof of this result is essentially the same as that for the higher dimensional situation given in [10]. We take this opportunity to supply a detailed proof of Lemma 3.1 which works in all dimensions and hence clarify a point in the corresponding argument in [10]. As a corollary, we find a large number of conformal classes with positive Paneitz operators.

Corollary 1.2. There exist conformal structures on the connected sum of a finite number of copies of $S^{1} \times S^{2}$ for which the Paneitz operator is positive.

On the other hand, the Paneitz operator on the standard 3-sphere is not positive as its lowest eigenvalue is negative. Since the 3 -sphere is conformally the same as a long cylinder capped off at the ends by spherical caps, it is somewhat surprising that the operator should pick up a negative eigenvalue. To understand this situation, it is helpful to remark that contrary to the case of the Yamabe equation, the energy integrand (1.5) is not pointwise conformally covariant, it is only so after integration by parts. The boundary term thus becomes important in any gluing construction. Since the class of conformal structures with positive conformal Laplacian and positive Paneitz operators do not contain the most singular case of the standard 3 -sphere, it is possible to solve the equation to prescribe the $Q$-curvature in this case.

Theorem 1.3. If $(M, g)$ is a three dimensional closed manifold such that the Paneitz operator $P$ is positive, then the equation (1.4) has a positive solution with $Q_{\bar{g}}$ being a negative constant.

Our approach to this problem is variational. We consider the functional $Q[u]=\left(\int_{M} u^{-6} \mathrm{~d} v\right)^{1 / 3} \int_{M} P u \cdot u \mathrm{~d} v$. Due to the presence of the negative power nonlinearity, it is not possible to localize the analysis. The analysis of $Q[u]$ presents a number of features that are distinct from that of the Yamabe quotient. The presence of the negative exponent term means that the analysis is centered on preventing the conformal factor from touching zero. Fortunately in the case under consideration, there is compactness in the minimizing sequence of the energy functional. Thus as a corollary of the proof we obtain an Sobolev type inequality of the form:

$$
0<Q_{p}[M]=\inf \left(\int_{M} u^{-p} \mathrm{~d} v\right)^{2 / p} \int_{M} P u \cdot u \mathrm{~d} v, 1<p<\infty .
$$

The key fact we need (Lem. 4.3) is motivated by the classification of entire solutions on $R^{3}$ of the equation $\Delta^{2} u=-u^{-7}$ which appears in [5]. In the case of the standard 3-sphere, it is possible to determine the best constant in (1.6) for the standard 3-sphere with a negative $Q_{6}$. However there are other issues to be resolved in the more general situation. We hope to return to this question on a later occasion.

Finally we outline the paper. In Section 2 we set the notations as well as some elementary examples of Paneitz operators. In Section 3 we prove Theorem 1.1. In Section 4 we prove Theorem 1.3. 


\section{Notations AND EXAmples}

In this section we compute a few examples of the Paneitz operator.

$$
P=(-\Delta)^{2}+\delta\left(\frac{5}{4} R g-4 R i c\right) d-\frac{1}{2} Q
$$

where

$$
Q=-2|R i c|^{2}+\frac{23}{32} R^{2}-\frac{1}{4} \Delta R
$$

For the cylinder, which is conformally equivalent to the punctured 3 -space $\mathcal{R}^{3}-\{0\}$, we write $x=|x| \cdot \frac{x}{|x|}=\mathrm{e}^{t} \cdot \sigma$ with $|x|=\mathrm{e}^{t}$ and $\sigma=\frac{x}{|x|} \in S^{2}$. The metric is given by $g=\frac{|\mathrm{d} x|^{2}}{|x|^{2}}=\mathrm{d} t^{2}+\mathrm{d} \sigma^{2}$ where $\mathrm{d} \sigma^{2}$ the canonical metric on the 2 -sphere. We have $R=2,|R i c|^{2}=2, Q=-\frac{9}{8}$. With the notation $\nabla^{T}, \Delta^{T}$ denoting gradient and Laplacian in the directions tangential to the 2 -sphere, we find

$$
P=\left(\partial_{t}^{2}+\Delta^{T}\right)^{2}-(5 / 2) \Delta+4 \Delta^{T}+\frac{9}{16}
$$

For functions $u$ with compact support on the cylinder, we find upon integrating by parts,

$$
\int P u \cdot u \mathrm{~d} v=\int\left[\left|u^{\prime \prime}\right|^{2}+(5 / 2)\left|u^{\prime}\right|^{2}+\left|\Delta^{T} u\right|^{2}\right] \mathrm{d} v+\int\left[2\left|\nabla^{T} u^{\prime}\right|^{2}-(3 / 2)\left|\nabla^{T} u\right|^{2}+\frac{9}{16} u^{2}\right] \mathrm{d} v
$$

which is positive after integrating in the 2 -sphere direction.

For the standard 3 -sphere, we have $R c=2 g$, so that $R=6$, and $Q=\frac{15}{8}$.

$$
P=(-\Delta)^{2}+(1 / 2) \Delta-\frac{15}{16}
$$

To determine the base eigenvalue of the operator $P$, we rewrite the energy integral, using the Bochner formula,

$$
\int_{S^{3}} P u \cdot u \mathrm{~d} v=\int_{S^{3}}\left\{\left|\nabla^{2} u\right|^{2}+(3 / 2)|\nabla u|^{2}-\frac{15}{16} u^{2}\right\} \mathrm{d} v .
$$

It follows that the base eigenvalue of the operator $P$ is $-\frac{15}{16}$.

\section{Connected Sum}

In this section we show that the positivity of the Paneitz operator is preserved in taking a connected sum provided the gluing cylinder is sufficiently long and thin. Let $(M, g)$ and $\left(M^{\prime}, g^{\prime}\right)$ be compact 3-manifolds whose fourth order Paneitz operators are positive. Due to the conformal covariance property of the Paneitz operator, the operator will remain positive when $g$ is replaced by a conformal metric. From continuity consideration, we may assume that $M$ and $M^{\prime}$ contain sufficiently small balls $B$ and $B^{\prime}$ of radius $\epsilon$ in which the metrics $g$ and $g^{\prime}$ are conformally flat. By rescaling of the coordinates, we may assume these are balls of unit radius. In forming a connected sum, it is advantageous to use the cylindrical coordinates for $B: x=|x| \cdot \frac{x}{|x|}$, where we set $|x|=\mathrm{e}^{t}$ and $\sigma=\frac{x}{|x|}$. Likewise, for $B^{\prime}$ we have $x^{\prime}=\mathrm{e}^{t^{\prime}} \cdot \sigma^{\prime}$. We identify $(t, \sigma)$ with $\left(-L-t^{\prime}, \sigma^{\prime}\right)$ for $-L \leq t \leq 0$, and call $L$ the length parameter. We then glue the metrics together over the common region $-L \leq t \leq 0$. We may also suppose, using the conformal covariance property of the Paneitz operator that the original metrics $g$ and $g^{\prime}$ agree with the cylinder metric $\mathrm{d} t^{2}+\mathrm{d} \sigma^{2}$ over the common region $-L \leq t \leq 0$. 
To prove that the conformal structure on the connected sum has a positive Paneitz operator, we proceed by contradiction. Assume to the contrary, that there is a sequence of length parameters $L$ tending to infinity, for which the Paneitz operator $P_{L}$ on the connected sum has a nonpositive eigenvalue. We will show that there is a subsequence of eigenfunctions $u_{L}$ which converges on either $M$ or $M^{\prime}$ to a function $v$ for which $\int P v \cdot v \mathrm{~d} V \leq 0$. This will be a contradiction to our assumption.

To make use of the assumption that $(M, g)$ and $\left(M^{\prime}, g^{\prime}\right)$ have positive Paneitz operators, we will cap off the cylinder at various values of $t$ parameter and extend the eigenfunctions into the caps.

Lemma 3.1. Let $v$ be a smooth function in a neighborhood of the unit sphere $S^{2}$ in $\mathcal{R}^{3}$, let $V$ be the biharmonic function defined on the unit ball $B$ having the same boundary value and normal derivative as $v$ on $S^{2}$. Then there is a constant $C$ such that

$$
\int_{B}|\Delta V|^{2}+|\nabla V|^{2}+V^{2} \mathrm{~d} x \leq C\left\{\int_{S^{2}}\left|\Delta^{T} v\right|^{2}+|\nabla v|^{2}+v^{2} \mathrm{~d} \sigma\right\} .
$$

Proof. We begin with the representation formula for $V$ :

$$
V(x)=\int_{S^{2}}\left(\partial_{n} \Delta\right)_{y} G(x, y) v(y) \mathrm{d} \sigma(y)-\int_{S^{2}} \Delta_{y} G(x, y) \partial_{n} v(y) \mathrm{d} \sigma(y),
$$

where

$$
G(x, y)=-\frac{1}{4 \pi}\left\{|x-y|-|y||x-\bar{y}|+\frac{1}{2} \frac{\left(1-|x|^{2}\right)\left(1-|y|^{2}\right)}{|y||x-\bar{y}|}\right\}
$$

and $\bar{y}$ is the inversion of $y$ in the unit sphere $S^{2}=\{y|| y \mid=1\}$. Denote by $I$ the second integral in (3.2) and by $I I$ the first integral in (3.2), so that $V(x)=I+I I$. A direct calculation yields for the most singular term for $|y|=1$ :

$$
I=-\frac{1}{4 \pi} \int_{S^{2}}\left\{\frac{\left(1-|x|^{2}\right)^{2}}{|x-y|^{3}}\right\} \partial_{n} v(y) \mathrm{d} \sigma(y)
$$

and

$$
I I=\frac{1}{4 \pi} \int_{S^{2}}\left(1-|x|^{2}\right)\left\{\frac{6\left(|x|^{2}-y \cdot x\right)^{2}}{|x-y|^{5}}+\frac{2+3 y \cdot x-5|x|^{2}}{|x-y|^{3}}\right\} v(y) \mathrm{d} \sigma(y) .
$$

It will suffice to prove

$$
\int_{B}|\Delta V|^{2}+V^{2} \mathrm{~d} x \leq C\left\{\int_{S^{2}}\left|\nabla^{2} v\right|^{2}+|\nabla v|^{2}+v^{2} \mathrm{~d} \sigma\right\} .
$$

Let us consider the new function

$$
w(v)=\frac{1}{4 \pi}\left(1-|x|^{2}\right) \int_{S^{2}} \frac{v}{|x-y|^{3}} \mathrm{~d} \sigma(y),
$$

for any given continuous function $v$ on $S^{2}$. 
It is clear from Poisson integral formula, $w(v)$ is a harmonic function in $B^{3}$. We recall that for harmonic functions $h$ :

$$
\int_{B} h^{2} \mathrm{~d} x \leq \int_{S^{2}} h^{2} \mathrm{~d} \sigma
$$

Apply this to $w$, keep in mind that $w=v$ on the unit sphere $S^{2}$ to get

$$
\int_{B} w^{2} \mathrm{~d} x \leq \int_{S^{2}} v^{2} \mathrm{~d} \sigma
$$

To bound the first integral, we observe that $1-|x|^{2} \leq 1$, thus

$$
|I| \leq C \frac{1-|x|^{2}}{4 \pi} \int_{S^{2}} \frac{|\nabla v(y)|}{|x-y|^{3}} \mathrm{~d} \sigma(y) \leq C w(|\nabla v|) .
$$

Similarly,

$$
\mid \text { the second integral of } I I \mid \leq C \frac{1-|x|^{2}}{4 \pi} \int_{S^{2}} \frac{|v|}{|x-y|^{3}} \mathrm{~d} \sigma(y) \leq C w(|v|) \text {. }
$$

and now since $\left.|| x\right|^{2}-x \cdot y|=| x \cdot(x-y)|\leq| x|| x-y \mid$;

$$
\mid \text { the first integral of } I I \mid \leq C \frac{1-|x|^{2}}{4 \pi} \int_{S^{2}} \frac{|v|}{|x-y|^{3}} \mathrm{~d} \sigma(y) \leq C w(|v|) \text {. }
$$

Then we square $V$ and integrate over $B$ and apply (3.7) to find,

$$
\int_{B} V^{2} \mathrm{~d} x \leq C \int_{S^{2}}|\nabla v|^{2}+v^{2} \mathrm{~d} \sigma
$$

To bound the integral $\int_{B}|\Delta V|^{2} \mathrm{~d} x$, we apply the inequality (3.7) to $\Delta V$ to obtain

$$
\int_{B}|\Delta V|^{2} \mathrm{~d} x \leq \int_{S^{2}}|\Delta V|^{2} \mathrm{~d} \sigma
$$

The desired bound then follows from the following explicit formula [10] for $\Delta u$ in terms of the intrinsic Laplacian $\Delta^{T}$ operating on the boundary data $\phi=\partial_{n} u$ and $\psi=u$ that can be derived via a calculation utilizing the spherical harmonics.

$$
\Delta u=2\left(\sqrt{-\Delta^{T}+\frac{1}{4}}+1\right) \phi+\left\{2 \Delta^{T}-\left(\sqrt{-\Delta^{T}+\frac{1}{4}}-\frac{1}{2}\right)\right\} \psi
$$

This finishes the proof of Lemma 3.1.

To apply Lemma 3.1, we split the manifold $M$ into $M_{0}=M-B$ and $B$, and likewise $M^{\prime}$ into $M_{0}^{\prime}=M^{\prime}-B^{\prime}$ and $B^{\prime}$ so that $M \# N=M_{0} \cup M_{0}^{\prime} \cup[0, L] \times S^{2}$. When we cap off $M_{0} \cup[0, t] \times S^{2}$ by attaching $B$ to the boundary $\{t\} \times S^{2}$ we view the resulting manifold as conformally the same as $M$, and we extend the boundary data of the eigenfunction $u_{L}$ to a biharmonic function on $B$. (More precisely, in switching over to a conformal metric, we need to use the conformal covariance property of the operators to transform the relevant boundary data.) We denote the resulting function on $M$ by $v_{L}$. Coming from the other end, we view $M^{\prime}$ as $M_{0}^{\prime} \cup[t, L] \times S^{2}$, and extend the boundary data of the function $u_{L}$ to $M^{\prime}$ and denote the resulting function on $M^{\prime}$ by $v_{L}^{\prime}$. 
To account for the energy of the several functions we have

$$
E(u)=\int P u u \mathrm{~d} V=\int e(u) \mathrm{d} V
$$

where

$$
e(u)=|\Delta u|^{2}+\frac{5}{4} R|\nabla u|^{2}-4 R c(\nabla u, \nabla u)-\frac{1}{2} Q u^{2} .
$$

The positivity assumption on $M$ and Lemma 3.1 imply

$$
\int_{M_{0}} e(u) \mathrm{d} V+\int_{0}^{t} \int_{S^{2}} e(u(s, \sigma)) \mathrm{d} \sigma \mathrm{d} s+C_{n} \int_{S^{2}} e(u(t, \sigma)) \mathrm{d} \sigma>0 .
$$

Similarly,

$$
\int_{M_{0}^{\prime}} e(u) \mathrm{d} V+\int_{t}^{L} \int_{S^{2}} e(u(s, \sigma)) \mathrm{d} \sigma \mathrm{d} s+C_{n} \int_{S^{2}} e(u(t, \sigma)) \mathrm{d} \sigma>0 .
$$

The nonpositivity assumption on $M \# N$ implies that

$$
\int_{M_{0}} e(u) \mathrm{d} V+\int_{M_{0}^{\prime}} e(u) \mathrm{d} V+\int_{0}^{L} \int_{S^{2}} e(u(s, \sigma)) \mathrm{d} \sigma \mathrm{d} s \leq 0 .
$$

Since on the tube $[0, L] \times S^{2}$ the energy integral $\int_{S^{2}} e(u(s, \sigma)) \mathrm{d} \sigma$ is positive for each $0<s<L$, we observe that at least one of the numbers: $\int_{M_{0}} e(u) \mathrm{d} v$ and $\int_{M_{0}^{\prime}} e(u) \mathrm{d} v$ is negative. Hence there are numbers $0<t_{0} \leq t_{1}<L$ so that

$$
\int_{M_{0}} e(u) \mathrm{d} V+\int_{0}^{t_{1}} \int_{S^{2}} e(u(s, \sigma)) \mathrm{d} \sigma \mathrm{d} s=0
$$

and

$$
\int_{M_{0}^{\prime}} e(u) \mathrm{d} V+\int_{t_{0}}^{L} \int_{S^{2}} e(u(s, \sigma)) \mathrm{d} \sigma \mathrm{d} s=0 .
$$

In case the lowest eigenvalue of the Paneitz on $M \# M^{\prime}$ is zero, and $u$ the corresponding eigenfunction, we have $t_{0}=t_{1}$. Since

$$
-\int_{t_{0}}^{t_{1}} \int_{S^{2}} e(u(s, \sigma)) \mathrm{d} \sigma \mathrm{d} s=\int_{M_{0}} e(u) \mathrm{d} V+\int_{M_{0}^{\prime}} e(u) \mathrm{d} V+\int_{0}^{L} \int_{S^{2}} e(u(s, \sigma)) \mathrm{d} \sigma \mathrm{d} s .
$$

We can rewrite (3.12) and (3.13) by using (3.15) and (3.16) as

$$
C_{n} \int_{S^{2}} e(u(t, \sigma)) \mathrm{d} \sigma>\int_{t}^{t_{1}} \int_{S^{2}} e(u(s, \sigma)) \mathrm{d} \sigma \mathrm{d} s \text { for } 0 \leq t \leq t_{1},
$$

and

$$
C_{n} \int_{S^{2}} e(u(t, \sigma)) \mathrm{d} \sigma>\int_{t_{0}}^{t} \int_{S^{2}} e(u(s, \sigma)) \mathrm{d} \sigma \mathrm{d} s \quad \text { for } t_{0} \leq t \leq L .
$$


Consequently, we find

$$
2 C_{n} \int_{S^{2}} e(u(t, \sigma)) \mathrm{d} \sigma \geq \int_{t_{0}}^{t_{1}} \int_{S^{2}} e(u(s, \sigma)) \mathrm{d} \sigma \mathrm{d} s \quad \text { for } t_{0} \leq t \leq t_{1} .
$$

Upon integration we find

$$
2 C_{n} \geq\left(t_{1}-t_{0}\right)
$$

Hence the middle interval is bounded independent of $L$. So as $L$ tends to infinity we have at least one of $t_{1}$ and $L-t_{0}$ tends to infinity. Further, on one of these long tubes, equations (3.17) or (3.18) implies exponential decay of the energy on the $t$ slices. A subsequence of the eigenfunctions $u_{L}$ then converges uniformly on compact subsets of $M_{0} \cup[0, \infty) \times S^{2}$ or $M_{0}^{\prime} \cup[0, \infty) \times S^{2}$ to an eigenfunction $u_{\infty}$ which has exponential decay in the tube. The conformal transform of this function to $\tilde{u}_{\infty}$ on $M$ or $M^{\prime}$ is then a function with $W^{2,2}$ norm bounded but satisfies the condition

$$
\int e\left(\tilde{u}_{\infty}\right) \mathrm{d} V \leq 0
$$

This is a contradiction to our assumption. Thus we have proved the Theorem 1.1.

\section{Proof of Theorem 1.3}

We will consider the variational problem to minimize the functional

$$
F_{6}(u)=\left(\int_{M} u^{-6} \mathrm{~d} v\right)^{1 / 3} \int_{M} P u \cdot u \mathrm{~d} v
$$

The critical points of the functional $F_{6}$ satisfy the equation (1.4) with $Q_{\bar{g}}$ given by a constant. The functional $F_{6}$ is invariant under scaling, and more generally, invariant under the action induced by conformal transformations $T$ of the manifold $M$. To break this natural symmetry, we will consider the more general functional

$$
F_{p}(u)=\left(\int_{M}|u|^{-p} \mathrm{~d} v\right)^{2 / p} \int_{M} P u \cdot u \mathrm{~d} v
$$

for every $p \geq 6$ over the space $H^{2,2}(M)$.

For simplicity, we denote

$$
H_{p}=\left\{u \in H^{2,2}(M): \int_{M}|u|^{-p} \mathrm{~d} v=1\right\}
$$

and assume that $\int_{M} \mathrm{~d} v=1$.

Lemma 4.1. There exists a positive smooth function which minimizes the functional $F_{p}(u)$ over $H_{p}$ if $p>6$.

Proof. Since $P$ is positive, there exists a positive constant $\lambda>0$ such that for every $u \in H_{p}$,

$$
\int_{M} P u \cdot u \mathrm{~d} v \geq \lambda \int_{M} u^{2} \mathrm{~d} v .
$$


Thus $F_{p}(u)$ has a lower bound over $H_{p}$ (nonnegative). Let $\left\{u_{k}\right\}$ be a minimizing sequence of $F_{p}(u)$ over $H_{p}$. First of all, since $u_{k} \in H_{p}, \int_{M}\left|u_{k}\right|^{-p} \mathrm{~d} v=1$. Thus for every $k$,

$$
\int_{M} u_{k}^{2} \mathrm{~d} v \leq \frac{1}{\lambda} \int_{M} P u_{k} \cdot u_{k} \mathrm{~d} v=\frac{1}{\lambda} F_{p}\left(u_{k}\right) \leq C
$$

for some constant $C$ since $\left\{u_{k}\right\}$ is a minimizing sequence.

From $F_{p}\left(u_{k}\right) \leq C$ and the definition of the Paneitz operator, we have the following estimates:

$$
\begin{aligned}
\int_{M}\left(\Delta u_{k}\right)^{2} \mathrm{~d} v & =F_{p}\left(u_{k}\right)-\frac{5}{4} \int_{M} R\left|\nabla u_{k}\right|^{2} \mathrm{~d} v+4 \int_{M} R i c\left(\nabla u_{k}, \nabla u_{k}\right) \mathrm{d} v+\frac{1}{2} \int_{M} Q u_{k}^{2} \mathrm{~d} v \\
& \leq\left[\frac{5}{4} \max |R|+4 \max |R i c|\right] \int_{M}\left|\nabla u_{k}\right|^{2}+\frac{1}{2} \max |Q| \int_{M} u_{k}^{2} \mathrm{~d} v+C \\
& \leq \frac{1}{2} \int_{M}\left(\Delta u_{k}\right)^{2} \mathrm{~d} v+C_{1} \int_{M} u_{k}^{2} \mathrm{~d} v+C .
\end{aligned}
$$

Clearly from this and (4.5), we conclude that $\left\{u_{k}\right\}$ is a bounded sequence in $H^{2,2}(M)$. Thus it is standard that there exists a subsequence, still denoted by $\left\{u_{k}\right\}$, such that $u_{k}$ weakly converges to some function $u_{p} \in H^{2,2}(M)$. As a consequence, $u_{k}$ converges to $u_{p}$ strongly in $H^{1,2}(M)$ and almost everywhere on $M$.

Now let $\alpha=\frac{p}{3}-1>1$. It follows from $\int_{M}\left(\Delta u_{k}\right)^{2} \mathrm{~d} v \leq C, \int_{M}\left|\nabla u_{k}\right|^{2} \mathrm{~d} v \leq C$ and Sobolev's inequality that

$$
\int_{M}\left|\nabla u_{k}\right|^{6} \mathrm{~d} v \leq C
$$

Hence by Hölder inequality, we have

$$
\int_{M}\left|\nabla\left(\frac{1}{\left|u_{k}\right|^{\alpha}}\right)\right|^{2} \mathrm{~d} v=\alpha^{2} \int_{M} \frac{\left.|\nabla| u_{k}\right|^{2}}{\left|u_{k}\right|^{2(\alpha+1)}} \leq\left(\int_{M}\left|\nabla u_{k}\right|^{6} \mathrm{~d} v\right)^{1 / 3} \cdot\left(\int_{M}\left|u_{k}\right|^{-3(\alpha+1)} \mathrm{d} v\right)^{2 / 3} .
$$

Now the choice of $\alpha$ implies $3(\alpha+1)=p$. Thus we conclude from the fact that $\int_{M}\left|u_{k}\right|^{-p} \mathrm{~d} v=1$ that

$$
\int_{M}\left|\nabla \frac{1}{\left|u_{k}\right|^{\alpha}}\right|^{2} \mathrm{~d} v \leq C
$$

with $C$ depending only on the upper bound of $F_{p}$ and the geometry of $g$.

Also it follows from Hölder inequality that

$$
\int_{M}\left(\left|u_{k}\right|^{-\alpha}\right)^{2} \mathrm{~d} v \leq C
$$

for some constant $C$, again depending only on the same data as above.

The estimates (4.8) and (4.9) show that $\left\{\left|u_{k}\right|^{-\alpha}\right\}$ is a bounded sequence in $H^{1,2}(M)$. Thus there is a subsequence, again denoted by $\left\{u_{k}\right\}$ such that $\left|u_{k}\right|^{-\alpha}$ weakly converges to $\bar{u}_{p}^{-\alpha}$ in $H^{1,2}(M)$. However this implies the convergence holds almost everywhere on $M$. As we have shown that $u_{k}$ also converges to $u_{p}$ almost everywhere, $\left|u_{p}\right|=\bar{u}_{p}$ almost everywhere on $M$.

Now let $s=\frac{3 p}{p-3}$. The fact that $p>6$ implies $s<6$. Thus by the Rellich-Kondrachov compactness theorem, we get

$$
1=\lim _{k \rightarrow \infty} \int_{M}\left(\left|u_{k}\right|^{-\alpha}\right)^{s} \mathrm{~d} v=\int_{M}\left(\left|u_{p}\right|^{-\alpha}\right)^{s} \mathrm{~d} v
$$


Since $\alpha$ and $s$ are chosen so that $\alpha \cdot s=p$, we conclude that $u_{p} \in H_{p}$. Thus $u_{p}$ is a critical point of $F_{p}$ over $H_{p}$. Hence it weakly satisfies the equation

$$
P u_{p}=\lambda_{p}\left|u_{p}\right|^{-p} u_{p}^{-1}
$$

where $\lambda_{p}$ is the minimum value of $F_{p}$ over $H_{p}$. Since $u_{p}$ is not identically zero and $P$ is positive, $\lambda_{p}$ is positive. Since

$\left\|u_{p}\right\|_{H^{2,2}} \leq C$.

$$
\left\|u_{p}\right\|_{H^{2,2}} \leq \liminf _{k \rightarrow \infty}\left\|u_{k}\right\|_{H^{2,2}}
$$

We will use the equation to improve the regularity of the solution $u_{p}$. We begin with the $\left|\nabla u_{p}\right|$ is in $L^{6}(M)$ by Sobolev's embedding. Thus by the estimate (4.7), we know that if $\alpha=\alpha_{0}=(p-3) / 3>1$, then $\left.|\nabla| u_{p}\right|^{-\alpha} \mid$ is in $L^{2}(M)$. It follows that $\left|u_{p}\right|^{-\alpha}$ is in $L^{6}(M)$. Notice that $6 \alpha=2(p-3)>p$ if $p>6$. Then in (4.7), we choose $\alpha=\alpha_{1}=2 \alpha_{0}-1$, then by Sobolev's Embedding, we conclude that $\left|u_{p}\right|^{-\alpha_{1}}$ is in $L^{6}(M)$. Repeating this process, define

$$
\alpha_{n+1}=2 \alpha_{n}-1=2^{n}\left(\alpha_{0}-1\right)+1,
$$

to find that for any $n \geq 0,\left|u_{p}\right|^{-\alpha_{n}}$ is in $L^{6}(M)$. It is clear that $\alpha_{n}$ goes to infinity as $n$ goes to infinity. Hence we can choose $n$ such that $6 \alpha_{n} \geq 2(p+1)$.

What we have shown in previous paragraph is that the right hand side of equation (4.11) is in $L^{2}(M)$. Then it follows that $\left(\left|\Delta^{2} u\right|\right)^{2}$ is integrable. Thus $\Delta u_{p}$ is a continuous function on $M$ and bounded on $M$ in absolute value. It follows from

$$
\Delta\left(\frac{1}{u_{p}}\right)=-\frac{\Delta u_{p}}{u_{p}^{2}}+2 \frac{\left|\nabla u_{p}\right|^{2}}{u_{p}^{3}}
$$

that $\Delta\left(u_{p}^{-1}\right)$ is in $L^{2}(M)$. Hence $u_{p}^{-1}$ is a continuous function on $M$. Thus $u_{p}$ can never take zero value. Without loss of generality, we can assume that $u_{p}>0$, since otherwise we can take $-u_{p}$ as our solution. It will follow easily that $u_{p}$ is a $C^{\infty}$ smooth function. Therefore we have completed the proof of Lemma 4.1.

Lemma 4.2. Let $\lambda_{p}=\min _{u \in H_{p}} F_{p}(u)$ for $p \geq 6$. Then

$$
\lim _{p \rightarrow 6} \lambda_{p}=\lambda_{6}
$$

Proof. Since

let $\left\{u_{i}\right\}$ be a sequence in $H_{6}$ such that

$$
\lambda_{6}=\inf _{u \in H_{6}} F_{6}(u),
$$

as $i \rightarrow \infty$. For each fixed $i$, we have

$$
F_{6}\left(u_{i}\right) \rightarrow \lambda_{6}
$$

as $p \rightarrow 6$. It follows that

$$
\lambda_{p} \leq F_{p}\left(u_{i}\right) \rightarrow F_{6}\left(u_{i}\right)
$$

On the other hand, by Hölder inequality, we have

$$
F_{6}\left(u_{p}\right)=F_{p}\left(u_{p}\right) \cdot \frac{\left\|u_{p}^{-1}\right\|_{6}^{2}}{\left\|u_{p}^{-1}\right\|_{p}^{2}} \leq F_{p}\left(u_{p}\right)\left(\int_{M} \mathrm{~d} v\right)^{2\left(1-\frac{6}{p}\right)} .
$$

Thus it follows that

$$
\lambda_{6} \leq \lambda_{p}\left(\int_{M} \mathrm{~d} v\right)^{2\left(1-\frac{6}{p}\right)}
$$


Hence, we get

$$
\lambda_{6} \leq \underline{\lim }_{p \rightarrow 6} \lambda_{p}\left(\int_{M} \mathrm{~d} v\right)^{2\left(1-\frac{6}{p}\right)}=\underline{\lim }_{p \rightarrow 6} \lambda_{p}
$$

Therefore

$$
\lim _{p \rightarrow 6} \lambda_{p}=\lambda_{6}
$$

This finishes the proof of Lemma 4.2.

Lemma 4.3. There exists no $C^{4}$ function $v$ satisfying

(1) $\int_{\mathcal{R}^{3}} v^{-6} \mathrm{~d} v<\infty$;

(2) $v \geq 1$ and $v(0)=1$;

(3) $\int_{\mathcal{R}^{3}}(\Delta v)^{2} \mathrm{~d} v<\infty$;

(4) $\Delta^{2} v=v^{-7}$.

Proof. It follows from conditions (1) and (2) that for any $q \geq 6$,

$$
\int_{\mathcal{R}^{3}} v^{-q} \mathrm{~d} v<\infty
$$

Define

$$
w(x)=\frac{1}{4 \pi} \int_{\mathcal{R}^{3}} \frac{v^{-7}(y)}{|x-y|} \mathrm{d} y .
$$

Then for any $q>1, w(x)$ is in $L^{q}\left(\mathcal{R}^{3}\right)$ and

$$
\Delta w+v^{-7}=0
$$

For any $r>0$ and $x \in \mathcal{R}^{3}$, integrate condition (4) over the ball $B_{r}(x)$ to get

$$
\int_{B_{r}(x)} v^{-7} \mathrm{~d} x=\int_{B_{r}(x)} \Delta^{2} v \mathrm{~d} x=r^{2} \frac{\partial}{\partial r}\left[r^{-2} \int_{\partial B_{r}(x)} \Delta v \mathrm{~d} \sigma\right]
$$

Multiply $r^{-2}$ on both sides of (4.15) and integral from 0 to $r$ to have

$$
\int_{0}^{r} t^{-2} \int_{B_{t}(x)} v^{-7} \mathrm{~d} y \mathrm{~d} t=r^{-2} \int_{\partial B_{r}(x)} \Delta v \mathrm{~d} \sigma-\omega_{3}(\Delta v)(x) .
$$

Now multiply $r^{2}$ on both sides of (4.16)and integrate the resulting equation from 0 to $r$ to get

$$
\begin{aligned}
\int_{0}^{r} s^{2}\left[\int_{0}^{s} t^{-2} \int_{B_{t}(x)} v^{-7} \mathrm{~d} y \mathrm{~d} t\right] \mathrm{d} s & =\int_{B_{r}(x)} \Delta v \mathrm{~d} x-\omega_{3}(\Delta v)(x) r^{3} / 3 \\
& =r^{2} \frac{\partial}{\partial r}\left[r^{-2} \int_{\partial B_{r}(x)} v \mathrm{~d} \sigma\right]-\omega_{3}(\Delta v)(x) r^{3} / 3
\end{aligned}
$$


Finally multiply both sides of (4.17) by $r^{-2}$ and integrate the resulting equation from 0 to $R$ to get

$$
\begin{aligned}
g(R) & :=\int_{0}^{R} r^{-2}\left\{\int_{0}^{r} s^{2}\left[\int_{0}^{s} t^{-2} \int_{B_{t}(x)} v^{-7} \mathrm{~d} y \mathrm{~d} t\right] \mathrm{d} s\right\} \mathrm{d} r \\
& =R^{-2} \int_{\partial B_{R}(x)} v \mathrm{~d} \sigma-\omega_{3} v(x)-\omega_{3}\left(R^{2} / 6\right)(\Delta v)(x) .
\end{aligned}
$$

This is known as the mean value property for biharmonic function. By using L'Hospital's rule, we can see that

$$
\lim _{R \rightarrow \infty} \frac{g(R)}{R^{2}}
$$

exists and is less than a constant independent of $x$. Hence we can conclude that

$$
\Delta v(x)+C \geq 0
$$

for all $x \in \mathcal{R}^{3}$.

Notice that condition (4) and equation (4.14) imply that

$$
\Delta(\Delta v+w)=0 .
$$

Clearly $w$ is nonnegative and by equation (4.19), we see that $w+\Delta v$ is bounded from below, hence Liouville's theorem implies that $w+\Delta v$ is a constant:

$$
\Delta v=C-w .
$$

As pointed out earlier, $w$ is in $L^{q}\left(\mathcal{R}^{3}\right)$ for every $q>1$. Thus as $|x| \rightarrow \infty, w(x) \rightarrow 0$. This, together with the condition (3), we can see that the constant $C$ must be zero.

It follows from the definition of $w$ that $\Delta v$ is nonpositive. Notice that

$$
\Delta\left(\frac{1}{v}\right)=-\frac{\Delta v}{v^{2}}+2 \frac{|\nabla v|^{2}}{v^{3}} \geq 0 .
$$

It follows that $\frac{1}{v}$ must be a constant. Clearly this, together with condition (1), implies that $v$ must be everywhere infinity, which is a contradiction to condition (2).

We have completed the proof of Lemma 4.3 .

Lemma 4.4. Let $\left\{u_{p}\right\}$ be the positive smooth function found in Lemma 4.1 for every $p>6$. Then there is a positive constant $c_{0}$, independent of $p$ such that

$$
u_{p} \geq c_{0}>0 .
$$

Proof. Assume such a $c_{0}$ does not exist. Thus there exists a subsequence $p_{k} \rightarrow 6, u_{k}=u_{p_{k}}, z_{k} \in M$ such that $u_{k}\left(z_{k}\right)=\min u_{k}:=m_{k} \rightarrow 0$. Since $M$ is compact, there exists a point $z_{0}$ such that $z_{k} \rightarrow z_{0}$ as $k \rightarrow \infty$. Take a normal coordinate at $z_{0}$. In this coordinate,

$$
g_{i j}(x)=\delta_{i j}+O\left(|x|^{2}\right), \quad \operatorname{det} g_{i j}(x)=1+O\left(|x|^{2}\right) .
$$

Now let $x_{k}$ be the coordinate of $z_{k}$. Then as $k \rightarrow \infty, x_{k} \rightarrow 0$. Notice that $u_{k}$ satisfies

$$
\Delta^{2} u_{k}-\delta\left[\frac{5}{4} R g-4 R i c\right] \mathrm{d} u_{k}-\frac{1}{2} Q u_{k}=\lambda_{k} u_{k}^{-(p+1)},
$$


where

and $\lambda_{k}=\lambda_{p_{k}}$.

$$
\Delta=\frac{1}{\sqrt{\operatorname{det} g}} \partial_{i}\left(\sqrt{\operatorname{det} g} g^{i j} \partial_{j}\right)
$$

Suppose this equation holds on the ball $|x|<1$. Define

$$
v_{k}(x)=m_{k}^{-1} u_{k}\left(\delta_{k} x+x_{k}\right),
$$

where $\delta_{k}=m_{k}^{\left(p_{k}+2\right) / 4} \rightarrow 0$ as $k \rightarrow \infty$. Thus $v_{k}$ is well defined on the ball $B_{\rho_{k}}(0)$ where $\rho_{k}=\left(1-\left|x_{k}\right|\right) / \delta_{k} \rightarrow \infty$ as $k \rightarrow \infty$. Denote $\sqrt{(\operatorname{det} g)\left(\delta_{k} x+x_{k}\right)}$ by $b_{k}$ and $g^{i j}\left(\delta_{k} x+x_{k}\right)$ by $a_{k}^{i j}$. In terms of $v_{k}$, they satisfy the equation:

$$
\begin{aligned}
\frac{1}{b_{k}} \frac{\partial}{\partial x_{i}}\left\{b_{k} a_{k}^{i j} \frac{\partial}{\partial x_{j}}\left[\frac{1}{b_{k}} \frac{\partial}{\partial x_{l}}\left(b_{k} a_{k}^{l m} \frac{\partial v_{k}}{\partial x_{m}}\right)\right]\right\}= & m_{k}^{-1} \delta_{k}^{4}\left(\Delta^{2} u_{k}\right)\left(\delta_{k} x+x_{k}\right) \\
= & m_{k}^{-1} \delta_{k}^{4}\left[\delta\left(\frac{5}{4} R g-4 R i c\right) \mathrm{d} u_{k}+\frac{1}{2} Q u_{k}+\lambda_{k} u_{k}^{-(p+1)}\right] \\
= & -\delta_{k}^{2} \frac{1}{b_{k}} \frac{\partial}{\partial x_{i}}\left[( b _ { k } a _ { k } ^ { i j } ) \left(\frac{5}{4} R\left(\delta_{k} x+x_{k}\right) g_{l j}\left(\delta_{k} x+x_{k}\right)\right.\right. \\
& \left.\left.-4 R_{l j}\left(\delta_{k} x+x_{k}\right)\right) a_{k}^{l m} \frac{\partial}{\partial x_{m}}\left(v_{k}\right)\right] \\
& +\frac{1}{2} Q\left(\delta_{k} x+x_{k}\right) \delta_{k}^{4} v_{k}+\lambda_{k} v_{k}^{-(p+1)} .
\end{aligned}
$$

On the other hand, the left hand side of the equation (4.25) can be written as

$$
\frac{1}{b_{k}} \frac{\partial}{\partial x_{\alpha}}\left[b_{k}^{2} a_{k}^{\alpha \beta} \frac{\partial}{\partial x_{\beta}}\left(\frac{1}{b_{k}}\right) a_{k}^{\gamma \eta} \frac{\partial}{\partial x_{\eta}} v_{k}+a_{k}^{\alpha \beta} \frac{\partial}{\partial x_{\beta}} \frac{\partial}{\partial x_{\eta}}\left(a_{k}^{\gamma \eta} b_{k}\right) \frac{\partial}{\partial x_{\eta}} v_{k}+b_{k} a_{k}^{\alpha \beta} a_{k}^{\gamma \eta} \frac{\partial}{\partial x_{\beta}} \frac{\partial}{\partial x_{\gamma}} \frac{\partial}{\partial x_{\eta}} v_{k}\right] .
$$

Now first we notice that

$$
\begin{gathered}
a_{k}^{i j}=g^{i j}\left(\delta_{k} x+x_{k}\right) \rightarrow \delta^{i j} \\
b_{k}=\sqrt{\operatorname{det} g\left(\delta_{k} x+x_{k}\right)} \rightarrow 1 \\
\frac{1}{2} Q\left(\delta_{k} x+x_{k}\right) \delta_{k}^{4} \rightarrow 0,
\end{gathered}
$$

as $k \rightarrow \infty$ where the convergence is $C^{1}$ uniform on any bounded set in $\mathcal{R}^{3}$.

Also notice that in (4.26), $b_{k}^{2} a_{k}^{\alpha \beta} a_{k}^{\gamma \eta} \partial_{\beta}\left(b_{k}^{-1}\right)$ and $a_{k}^{\alpha \beta} \partial_{\beta} \partial_{\eta}\left(a_{k}^{\gamma \eta} b_{k}\right)$ converge to 0 in $C^{1}$ norm uniformly.

Now since $v_{k}(x) \geq v_{k}(0)=1$, by $L^{q}$ and Schauder estimates for $v_{k}$ from equation (4.25), we will be able to conclude that for any $R>0$, there exist $C(R)>0$ and $k(R)>0$ such that

$$
\left\|v_{k}\right\|_{C^{4, \alpha}\left(\bar{B}_{k}\right)} \leq C(R) \text {, for any } k \geq k(R) .
$$

Take a sequence $R_{n} \rightarrow \infty$. By the diagonal procedure, we can get a subsequence such that $v_{n} \rightarrow v \in C^{4}\left(\mathcal{R}^{3}\right)$. This convergence is $C^{4}$ convergent on every $\bar{B}_{R_{n}}$. Then from equation (4.25), we get that $v$ satisfies the equation

$$
\Delta^{2} v=\lambda_{6} v^{-7}
$$


Without loss of generality, we can assume $\lambda_{6}=1$ since it is positive and multiplying $v$ by suitable constant will reduce the general case to this case.

Now by changing variables, we have

$$
\int_{|x|<(1 / 2) \delta_{k}^{-1}} v_{k}^{-p_{k}} b_{k} \mathrm{~d} x=\int_{B_{1 / 2}\left(x_{k}\right)} u_{k}^{-p_{k}} \sqrt{\operatorname{det} g} \mathrm{~d} x \cdot m_{k}^{\left(p_{k}-6\right) / 4} \leq\left\|u_{k}^{-1}\right\|_{p_{k}}^{p_{k}} m_{k}^{\left(p_{k}-6\right) / 4}=m_{k}^{\left(p_{k}-6\right) / 4} .
$$

Since $m_{k}^{\left(p_{k}-6\right) / 4}<1$ when $k$ large and $v_{k}^{-p_{k}} b_{k}$ uniformly converges to $v^{-6}$ on any bounded set. Thus by Fatou's lemma, we conclude that

$$
\int_{\mathcal{R}^{3}} v^{-6} \mathrm{~d} x \leq 1
$$

Now since $P$ is positive, it follows from equation for $u_{k}$ that

$$
\int_{M} u_{k}^{2} \mathrm{~d} v \leq \frac{\lambda_{p_{k}}}{\lambda} \int_{M} u_{k}^{-p_{k}} \mathrm{~d} v
$$

It implies that

$$
\int_{M}\left(\Delta u_{k}\right)^{2} \mathrm{~d} v \leq C
$$

for some constant independent of $k$.

Thus, similar to the argument for (4.33), it is not hard to see that

$$
\int_{\mathcal{R}^{3}}(\Delta v)^{2} \mathrm{~d} x<\infty
$$

Since every $v_{k} \geq 1$, thus $v \geq 1$. Now Lemma 4.3 implies that such $v$ does not exist. Thus we get a contradiction which allows us to conclude the proof of Lemma 4.4 .

Proof of Theorem 1.3. By Lemma 4.4, all solutions we have found in Lemma 4.1 are uniformly bounded from below as well as from above since $\Delta u_{p}$ are uniformally bounded in $L^{2}$. Thus the argument we have used in Lemma 4.1 will show that $u_{p}$ is uniformly bounded in $C^{k, \alpha}$ for any positive integer and any real number $0<\alpha<1$. Therefore there exists a subsequence $\left\{u_{p_{k}}\right\}$ such that $u_{p_{k}} \rightarrow u \in C^{\infty}(M)$ as $k \rightarrow \infty$ and $u>0$ will satisfy

$$
P u=\lambda_{6} u^{-7}
$$

This finishes the proof of Theorem 1.3.

\section{REFERENCES}

[1] M. Ahmedou, Z. Djadli and A. Malchiodi, Prescribing a fourth order conformal invariant on the standard sphere. Part I: Perturbation Result. Comm. Comtemporary Math. (to appear).

[2] T. Branson, Differential operators cannonically associated to a conformal structure. Math. Scand. 57 (1985) 293-345.

[3] A. Chang and P. Yang, Extremal metrics of zeta functional determinants on 4-manifolds. Ann. Math. 142 (1995) 171-212.

[4] A. Chang, M. Gursky and P. Yang, An equation of Monge-Ampere type in conformal geometry and four-manifolds of positive Ricci curvature. Ann. Math. (to appear)

[5] Y.S. Choi and X. Xu, Nonlinear biharmonic equation with negative exponent. Preprint (1999). 
6] Z. Djadli, E. Hebey and M. Ledoux, Paneitz operators and applications. Duke Math. J. 104 (2000) 129-169.

[7] C. Fefferman and R. Graham, Conformal Invariants, in Élie Cartan et les Mathématiques d'aujourd'hui. Asterisque (1985) 95-116.

[8] E. Hebey and F. Robert, Coercivity and Struwe's compactness for Paneitz type operators with constant coefficients. Preprint.

[9] S. Paneitz, A quartic conformally covariant differential operator for arbitrary pseudo-Riemannian manifolds. Preprint (1983).

[10] X. Xu and P. Yang, Positivity of Paneitz operators. Discrete Continuous Dynam. Syst. 7 (2001) 329-342. 